\title{
L'elaboratore elettronico nello studio delle serie temporali Filtraggio numerico ai minimi quadrati
}

\author{
(Electronic computer in the study of time series. \\ Numerical filtering to the last squares) \\ R. Pasquali tari - P. Randi $\left(^{*}\right)$
}

Ricevuto il 24 Gennaio 1972

Riassunto. - In questo lavoro viene discussa la opportunità di utilizzare il filtraggio numerico ai minimi quadrati nello studio delle serie temporali. Si dà la possibilita ai ricercatori di poter utilizzare questa tecnica anche senza specifiche competenze sulla programmazione di calcolatori elettronici. Il ricercatore può altresì ristrutturare a piacere una parte del programma descritto per introdurre condizioni che gli permettano di sfruttare completamente la sua serie di dati tenendo conto delle caratteristiche dei laboratori da cui essi dati provengono.

SUMMarY. - During this work we are concerned with the expedience to make use of numerical filtering to the last squares in the study of time series. The users can utilize this technique also without specific knowledge about programming of electronic computers. The users can also manipulate at pleasure a part of program in order to make the most of their data series, being known the features of the laboratories which the data come from.

\section{1. - TeCNica di Calcolo e possibilità.}

Il ricercatore può trovarsi nella condizione di avere una serie di dati molto grande e voler vederne le caratteristiche globali, ad esempio estraendo da essa informazioni relative alla esistenza di particolari bande di frequenza. Alcune tecniche numeriche relative a questa impostazione del problema, prendono il nome di filtraggio numerico $(2,3,4,6,7,8,9)$ e ne sono note di diverso tipo e con diverse finalità:

(*) Centro di Calcolo Interuniversitario dell'Italia Nord-Orientale, Casalecchio del Reno, Bologna (Italia). 
gli autori hanno scelto di effettuare il calcolo dei coefficienti del filtro numerico come descritto da (²) integrato da (1).

Je tecniche esposte permettono di estrarre bande di frequenza utilizanndo i coefficienti nel numero minore possibile e con una risposta in frequenza estremamente vicina a quella irleale; naturalmente essa ̀̀ in funzione del predetto numero di coefficienti.

Ar ogni morlo, poiché il problema a volte impone una separazione tra due tipi di informazioni altrimenti indistinguibili, la tecnica seguita dà quei coefficienti che sono stimati i migliori sotto le condizioni richieste.

le relazioni su cui si basa il calcolo dei coefficienti sono:

- per il filtro di tipo passa-basse frequenze sotto la condizione che la somma dei coefficienti sia 1 , si ha:

$g_{k}=\frac{1}{\pi}\left(\right.$ si $\pi \frac{2 k+1}{T}-s i \pi \frac{2 k-1}{T}-\frac{2}{2 N+1}$ si $\left.\pi \frac{2 N+1}{T}\right)+\frac{1}{2 N+1}$

- per il tipo di filtro passa-banda sotto la condizione che la somma dei coefficienti sia 0 , si ha:

$$
\begin{aligned}
g_{k} & =\frac{1}{\pi}\left(\operatorname{si\pi } \frac{2 k+1}{T}-s i \pi \frac{2 k+1}{t}-\operatorname{si} \pi \frac{2 k-1}{t}+\operatorname{si} \pi \frac{2 k-1}{T}\right)- \\
& -\frac{2}{\pi(2 N-1)}\left(\sin \frac{2 N+1}{t}-\operatorname{si\pi } \frac{2 N+1}{T}\right)
\end{aligned}
$$

- per il tipo di filtro passa-alte frequenze sotto la condizione che la somma dei coefficienti sia 0 , si ha:

$$
g_{0}=1-\frac{2}{\pi} \text { si } \frac{\pi}{T}+\frac{2}{(2 N+1) \pi} \text { si } \pi \frac{2 N+1}{T}-\frac{1}{2 N+1}
$$

e

$g_{k}=\frac{1}{\pi}\left(\right.$ si $\left.\eta \frac{2 k-1}{T}-s i \pi \frac{2 k+1}{T}\right)+\frac{2}{(2 k+1) \pi} \leqslant i \pi \frac{2 k+1}{T}-\frac{1}{2 N+1}$.

Per la discussione sulla scelta da imporre alla somma dei coefficienti si rimanda al lavoro $\left(^{2}\right)$ già citato.

Per rendere accessibile il programma di calcolo a qualsiasi ricercatore per qualsiasi problema di tale natura, gli autori hanno concentrato in poche scherle parametri tutte quelle informazioni che sono necessarie al calcolatore per la elaborazione dei dati. Vengono eseguiti in successione: calcolo dei coefficienti del filtro, risposta del filtro alle frequenze 
interessate, valutazione statistica dei dati filtrati sulla base della deviazione standard dei dati in ingresso, calcolo della serie residua e preparazione dei dati da graficare. La graficazione è ottenibile mediante un programma ${ }^{(5)}$ che fa parte della biblioteca del Centro di Calcolo.

I dati in ingresso vengono quindi trattati a seconda delle opzioni espresse dai ricercatori (v. descrizione delle schede parametri più sotto) sì da costituire un insieme omogeneo: le assenze di dati, inevitabili in lunghe registrazioni per mancanza di funzionamento delle apparecchiature $o$ alterazione delle stesse, possono essere o sostituite col valore della media della serie o con una interpolazione lineare fra gli estremi validi della interruzione (in questo caso la coincidenza fra il valore estremo finale calcolato e quello reale viene fissato dal ricercatore, in forma percentuale, a piacere), o con qualsiasi altra forma che il ricercatore ritenga opportuno. In questo ultimo easo, il ricercatore dovrà però scriversi una subroutine adatta col nome di CHIUS e con i parametri descritti più sotto che sostituisca quella inserita nella versione standard del programma.

I 6 parametri della subroutine CHIUS devono avere il seguente significato:

$P_{1}$ reale - valore dell'ultimo dato precedente al gap

$P_{2}$ reale - valore del primo dato successivo al gap

$P_{3}$ intero - numero d'ordine nella serie elaborata del dato il cui valore $\grave{\mathrm{e}} P_{1}$

$P_{4}$ intero - numero d'ordine nella serie elaborata del dato il cui valore $\grave{e} P_{2}$

$P_{5}$ reale - percentuale d'errore (vedi CHIUSX nella sezione "Utilizzazione del programma»)

$P_{6}$ reale - parametro di uscita: matrice dei dati da sostituire all'interno del gap (dimensione massima 500).

La subroutine viene chiamata all'interno del programma ogni qual volta si incontra un gap.

\section{2. - Descrizione tecilca del programma}

Il programma consta di un MAIN e di 5 subroutines, che sono: COEFIL, SINT, RDWR, CHIUS, MOVE. 
Il MAIN legge i parametri necessari alla elaborazione, li controlla e dispone le chiamate alle subroutines a seconda dei problemi che sono posti ed infine sovraintende alle stampe ed alla preparazione dei dati su nastro da utilizzarsi per la graficazione.

COEFIJ: calcola i coefficienti del filtro numerico con le morlalità richieste dal ricercatore; effettua il controllo sulla somma regli stessi $\left({ }^{2}\right)$ e calcola la risposta del filtro alle frequenze richieste.

SINT: è chiamata da COEFIL per il calcolo dei coefficienti del filtro numerico $\left.{ }^{(}\right)$.

RDWR: prepara i dati della serie temporale che debbono essere elaborati: elimina dati ritenuti non validi e provvede alla sostituzione degli stessi nelle forme previste.

CHIUS: viene chiamata da RDWR quando è richiesta una interpolazione lineare sostitutiva di dati mancanti o ritenuti non validi clal ricercatore. I'interpolazione è giurlicata ottima quando la differenza fra il primo dato valiclo, successivo ai rati mancanti, e quello calcolato dalla interpolazione sono uguali a meno del valore dichiarato dal ricercatore ( $\mathrm{v}$. "Utilizzazione del programma ").

MOVE: ripristina le condizioni di lavoro, mantenendo la sequenza temporale, in quanto il programma elabora una serie qualunque di dati, però a gruppi di 2000 per volta.

\section{3. - U'IILIZZazione DEL PROGRAMMa.}

Le schede contenenti i parametri e tutte le altre informazioni necessarie alla elaborazione della serie dei dati sono 7 e debbono essere sempre presenti.

Ise prime 4 sono relative al calcolo dei coefficienti del filtro e alla risposta del filtro alle frequenze di prova. Ise ultime 3 sono legate al processo di elaborazione dei lati.

Altre schede sono richieste se si verifica la condizione GAPIX $\neq 0$ come $\dot{e}$ scritto nella nota relativa a questo parametro nella $5^{\text {a }}$ scheda parametri. 
L'ELABORATORE ELETTRONICO NELLO STUDIO DELLE SERIE TEMPORALI, ETC. 67

1a scheda - descrizione del tipo di filtro.

Formato di lettura $(213,3$ F 10.4.)

\begin{tabular}{|c|c|c|}
\hline COLONNA & $\begin{array}{l}\text { NOME DELLA } \\
\text { VARIABILE }\end{array}$ & DESCRIZIONE \\
\hline $1-3$ & $\mathrm{KTF}$ & $\begin{array}{l}\text { se }=1 \text { filtro passa hasso } \\
\text { se }=2 \text { filtro passa alto }\end{array}$ \\
\hline $4-6$ & $\mathrm{KCOE}$ & $\begin{array}{l}\text { se }=3 \text { filtro passa banda } \\
\text { numero dei coeflicienti del filtro. Essendo } \\
\text { simmetrico, occorre indicare soltanto quanti } \\
\text { termini stanno da una parte rispetto al ter- } \\
\text { mine centrale; se si vuole un filtro di } 21 \text { ter- } \\
\text { mini KCOE sarà }=10 \text {. }\end{array}$ \\
\hline $7 \cdot 16$ & $\mathrm{TG}$ & valore del periodo su cui viene fatta la finestra. \\
\hline $17-26$ & $\mathrm{TP}$ & $\begin{array}{l}\text { valore del periodo a cui viene chiusa la fine- } \\
\text { stra (questo valore } \grave{\mathrm{e}} \neq 0 \text { solo quando } \mathrm{K} \mathrm{TF}=3 \text { ). }\end{array}$ \\
\hline $27-36$ & $S G$ & $\begin{array}{l}\text { valore a cui la somma dei coefficienti deve } \\
\text { essere uguagliata. } \\
\begin{array}{l}\text { In generale: } \\
\text { se } \mathrm{KTF}=2 \text { o } 3 \mathrm{SG}=0 \\
\text { se } \mathrm{KTF}=1 \quad \mathrm{SG}=1\end{array}\end{array}$ \\
\hline
\end{tabular}

$2^{\mathrm{a}}$ scheda - delinuta il campo dei test di fiducia.

Formato $=(I 3)$

\begin{tabular}{|c|c|c|}
\hline COLONNA & $\begin{array}{c}\text { NOME DELIA } \\
\text { VARIABILE }\end{array}$ & DEsCrizione \\
\cline { 2 - 3 } & NT & è il numero di periodi su cui si fa il test. \\
\hline
\end{tabular}


$3^{a}$ scheda - elenco di periodi su cui fare il test di fiducia.

Formato $=(8 \mathrm{E} 10.2)$

\begin{tabular}{|c|c|c|}
\hline COLONNA & $\begin{array}{c}\text { NOME DELLA } \\
\text { VARIABILE }\end{array}$ & DESCRIZIONE \\
\hline $1-n$ & $\mathrm{~T}$ & $\begin{array}{l}\text { valore dei periodi su cui si chiede il test } \\
\text { (questa scheda andrà ripetuta fino ad esauri- } \\
\text { mento dei periodi richiesti, mettendone } 8 \text { per } \\
\text { scheda). }\end{array}$ \\
\hline
\end{tabular}

$4^{a}$ scheda - chiude la prima serie di operazioni. Essa deve essere una scheda senza perforazioni.

$5^{\mathbf{a}}$ scheda - scelta dei tipi di calcolo e deviazione standard della serie.

Formato $=(\mathrm{I}, \mathrm{F} 10.0)$

\begin{tabular}{|c|c|c|c|}
\hline COLONNA & $\begin{array}{c}\text { NOME DELLA } \\
\text { VARIABILE }\end{array}$ & DeScRizione \\
\hline 1 & IPAR & $\begin{array}{l}s \theta=1 \text { viene calcolata solo la serie filtrata } \\
s \theta \neq 1 \text { viene calcolata anche la serie residua }\end{array}$ \\
$2-9$ & SIGMA & deviazione standard della serie originale. \\
\hline
\end{tabular}

$6^{\text {s }}$ scheda - contiene i parametri di lettura $\theta$ di elaborazione della serie. Formato $=(\mathrm{A3}, 2 \mathrm{I} 2, \mathrm{II}, \mathrm{IX}, 12,2 \mathrm{X}, 12,2 \mathrm{X}, \mathrm{I1}, \mathrm{IX}, \mathrm{F} 10.0$.

\begin{tabular}{|c|c|c|}
\hline COLONNA & $\begin{array}{c}\text { NOME DELLA } \\
\text { VARIABILE }\end{array}$ & DESCRIZIONE \\
\hline $1-3$ & IVISP & deve essere XXX \\
\hline 4-5 & $\mathrm{NN}$ & numero dei dati per record (max. 80 ) \\
\hline $6-7$ & M'T & $\begin{array}{l}\text { numero di unità logica di lettura dei dati } \\
\text { se }=5 \text { vengono lette schede } \\
\text { se }=66 \text { i dati sono su un filo di qualunque no- } \\
\text { me dichiarato sulla scheda LGo (v. } \\
\text { descrizione dell'INPUT più sotto) }\end{array}$ \\
\hline
\end{tabular}


Segue $6^{\mathrm{n}}$ scheda.

\begin{tabular}{|c|c|c|}
\hline COLONAA & $\begin{array}{c}\text { NOME DELLA } \\
\text { VARIABILE }\end{array}$ & DESCRIZIONE \\
\hline 8 & IZERX & $\begin{array}{ll}\text { se }=0 & \text { gli zeri della serie in INPUT sono } \\
& \text { reali } \\
\text { se } \neq 0 & \text { gli zeri (o l'assenza di perforazione) } \\
& \text { della serie in INPCT sono dati } \\
& \text { mancanti e verranno sostituiti nel } \\
& \text { corso del programma }\end{array}$ \\
\hline 10.11 & GAPIX $(*)$ & $\begin{aligned} \text { se }=0 & \text { non ci sono gaps in lettura } \\
\text { se } \neq 0 \text { indica il numero di volte che si } & \\
& \text { debbono saltare dati in lettura, dati } \\
& \text { che verranno sostituiti successiva- } \\
& \text { mente (max. 99) }\end{aligned}$ \\
\hline $14-15$ & $\operatorname{GAPEX}(*)$ & $\begin{array}{ll}\text { se }=0 \text { la successione dei dati ottenuta dopo } \\
\text { la lettura e l'eventuale sostituzione } \\
\text { (GAPIX } \neq 0 \text { ) è completa } \\
\text { se } \neq 0 \text { la successione ottenuta è incompleta. } \\
\text { se ad esempio GAPEX = l questo } \\
\text { significa che esiste un gruppo di } \\
\text { dati fisicamente mancanti; la serie } \\
\text { letta va integrata per ripristinare } \\
\text { la sequenza temporale }\end{array}$ \\
\hline 18 & CHIUSX & $\begin{array}{ll}\text { se }=0 & \text { nel gap viene inserita la media } \\
& \text { generale della serie } \\
\text { se } \neq 0 & \text { nel gap vengono inseriti i dati ela- } \\
& \text { borati mediante interpolazione li- } \\
& \text { neare usufruendo del metodo di } \\
& \text { chiusura }\end{array}$ \\
\hline $20-29$ & PERCX & $\begin{array}{l}\text { differenza percentuale richiesta dal metodo } \\
\text { di chiusura }\end{array}$ \\
\hline \multicolumn{3}{|c|}{$\begin{array}{l}(*) \text { N.B. - A questi due parametri è associato un certo numero di } \\
\text { schede che devono seguire la scheda parametri successiva a questa. } \\
\text { Su ciascuna scheda compaiono due valori interi, con formato }(2110) \text {, } \\
\text { rappresentanti il numero d'ordine, nella sequenza temporale da elabo- } \\
\text { rare, dei dati validi immediatamente esterni ad un gaps da integrare. } \\
\text { Relativamente ad un GAPIX }=1 \text { comparira una scheda contenente } \\
\text { ad esempio } 25 \text { nelle colonne } 9 \cdot 10 \text { e } 42 \text { nelle colonne } 19-20 \text {; questo signi- } \\
\text { fica che in lettura i dati dal } 26 . \text { mo al } 41 \text { li.mo non sono significativi e } \\
\text { debbono essere opportunamente sostituiti. Queste schede debbono } \\
\text { essere ordinate per valori screscenti dei limiti dei gaps. }\end{array}$} \\
\hline
\end{tabular}


$7^{a}$ scheda - contiene il formato di lettura dei dati. Formato $=(8 \mathrm{~A} 10)$

\begin{tabular}{|c|c|c|}
\hline COLON NA & $\begin{array}{c}\text { Nome DELla } \\
\text { VARIABILE }\end{array}$ & DESCRizione \\
\cline { 2 - 3 } & IFORM & $\begin{array}{l}\text { viene seritto il formato di lettura nel normale } \\
\text { linguaggio Fortran, comprese le parentesi } \\
\text { esterne }\end{array}$ \\
\hline
\end{tabular}

4. - Descrizione Dell'input E output.

Il programma è a disposizione dei ricercatori sotto forma di compilato ed è richiamabile mediante la seguente sequenza di scherle:

JOB.

REQUEST CIC1.

REWIND CIC1.

COPYN (A, LGO, CIC1)

LGO.

$7 / 8 / 9$

FILTRO, 6, CIC1

$7 / 8 / 9$

schede parametri e dati

$6 / 7 / 8 / 9$

L'uscita su tabulato è costituita dalle seguenti informazioni:

a) tipo di filtro e sue caratteristiche

b) coefficienti del filtro

c) test di fiducia

d) valori della serie filtrata

e) deviazione standard della serie filtrata

f) serie residua (se richiesta). 
L'EIABORATORE ELETTRONICO NELLO STUDIO DELLE SERIE TEMPORALI, ETC. 71

RISPOSTA DEI FILTRI

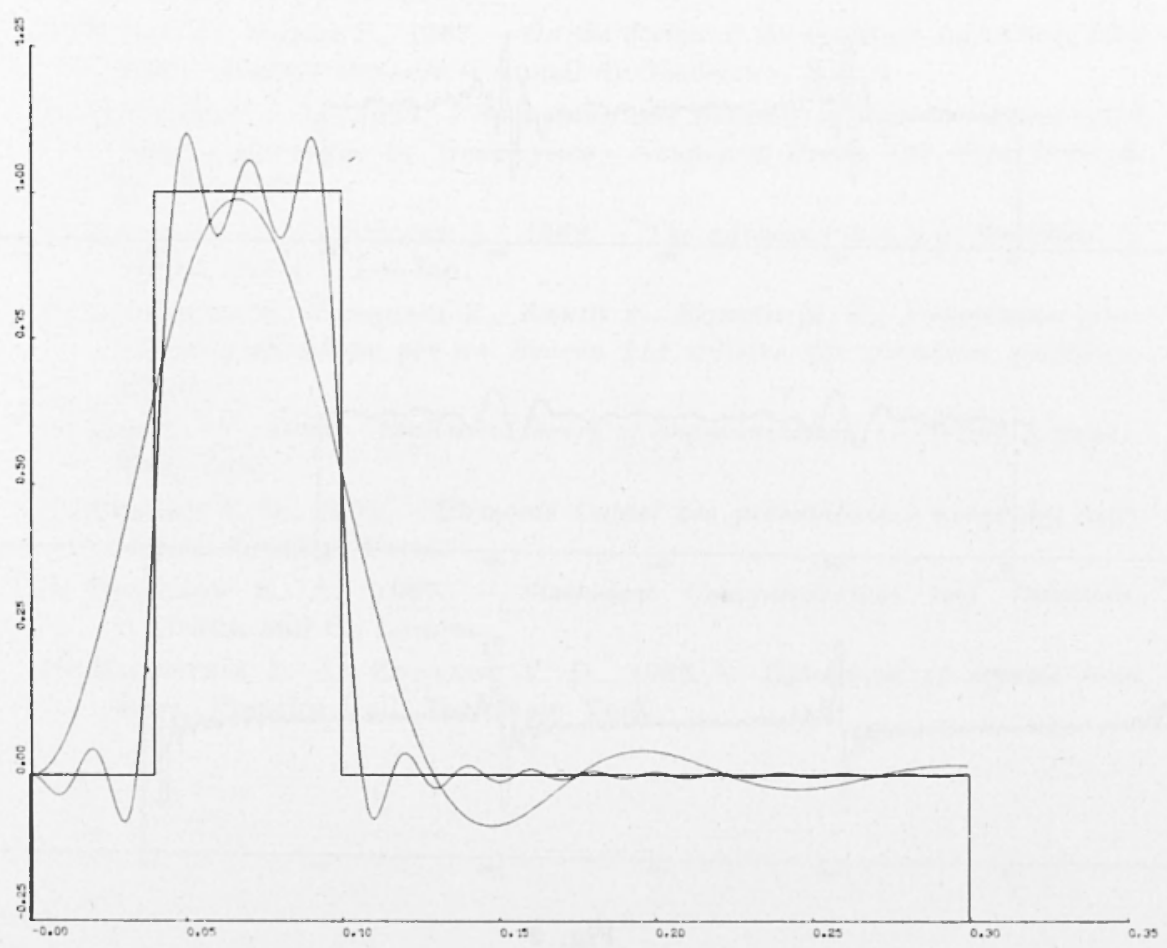

Fig. 1 
SERIE RESIDUA ， SERIE FILTRATA ， SERIE ORIGINALE
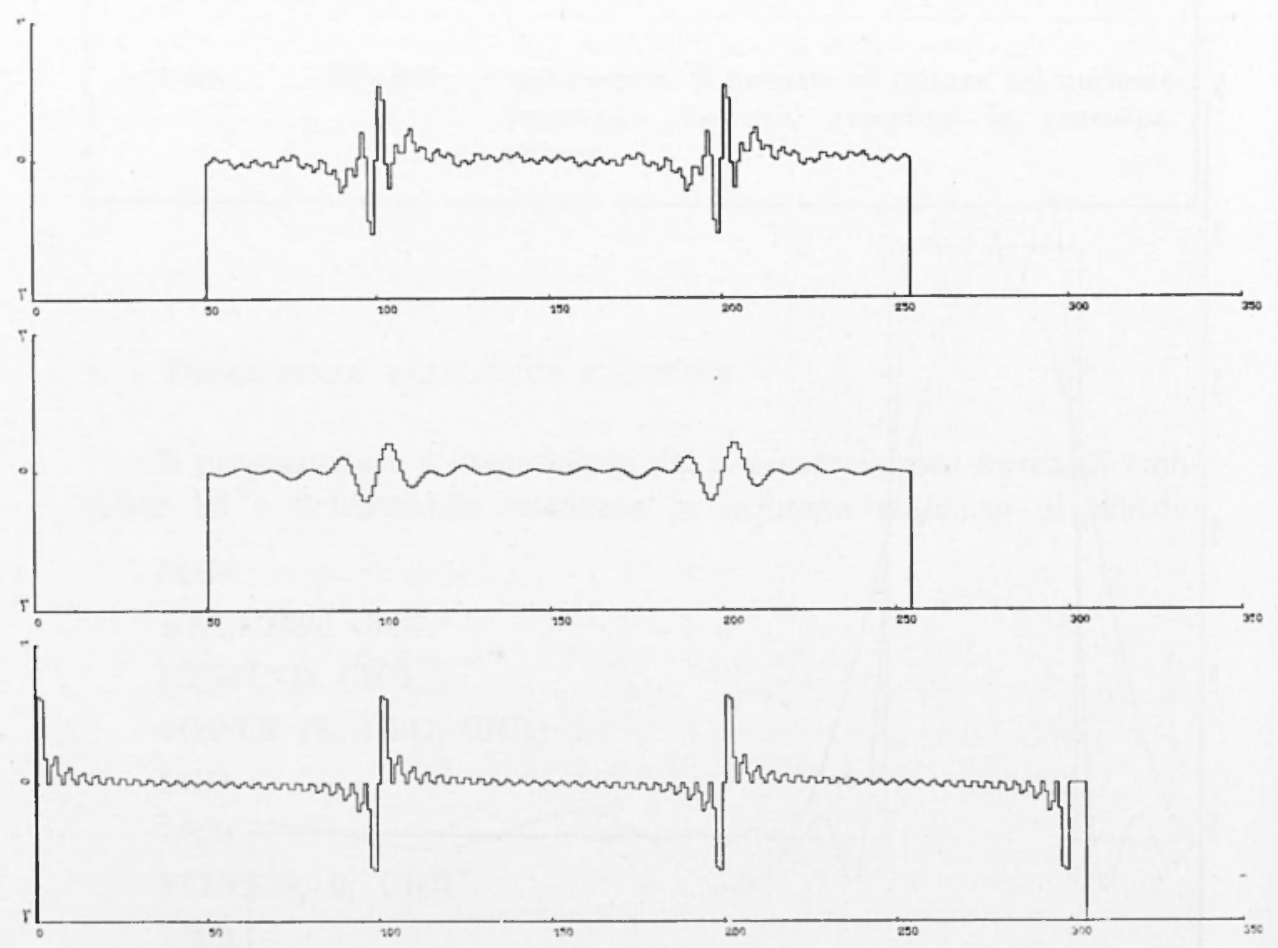

Fig. 2

Nelle figure 1 e 2 si mostrano risultati di elaborazioni ottenute utilizzando il lavoro già citato ${ }^{(5)}$. 


\section{BIBIIOGGRAFIA}

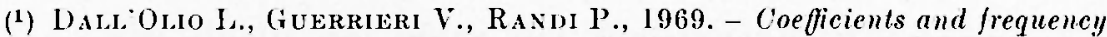
response computing for every numerical filter. "Lettere al Nuovo Cimento ", 1, pp. 271.278.

(2) GaLli II., RANDI P', 1967. - On the design of the optimum numerical filter with a preficed response. "Annali di Geofisica", XX, 4.

(3) Hol.owar .J. L., 1958. - Smoothing and filtering of time series and space fields. "Advances in Geophysics" Academic Press. Inc. New York, 4, pp. 351 -389.

(4) Kexide. M. G., Stuart A., 1969. - The advanced theory of Statistics. (. Griffin and C., London.

(5) Langarini M., Pasquali R., Raxjl P., Ronchil M. E., Programma generale di graficazione per un Benson 111 offlline (di prossima jubblicazione).

(') LEE Y. W., 1960. -- Statistical theory of communication. "J. Wiley \& Sons". New York.

(7) Perret B. G., 1964. - Éléments Calcul des probabilités à usage des ingénieurs. Eyrolles, Paris.

( ${ }^{8}$ Rominsox E. A., 1967. - Statistical Communication and Detection. C. Griffin and C., Idondon.

(9) Wansteis L. A., ZuBakov V. D., 1962. - Extraction of signals from noise. Prentice-Hall, Inc. New York. 